\title{
Tamaño del grupo, argumentación y lectura de tablas en estudiantes universitarios ${ }^{*}$
}

\author{
Group size, argumentation and table reading \\ in university students
}

\author{
Juan Manuel Curcio \\ ORCID: 0000-0001-8871-5315 \\ Nadia S. Peralta** \\ ORCID: 0000-0001-9950-6949 \\ Mariano Castellaro
ORCID: $0000-0001-5470-9662$
}

Instituto Rosario de Investigaciones en

Ciencias de la Educación (IRICE), Consejo Nacional de Investigaciones Científicas y Técnicas (CONICET), Universidad Nacional de Rosario, Argentina

Recibido: 18 de octubre de 2018 Revisado: 2 de diciembre de 2018 Aceptado: 4 de febrero de 2019

\section{Resumen}

La argumentación es una variable importante para entender el aprendizaje. Aunque hay varios artículos al respecto, su relación con el tamaño del grupo es una novedad. Se analiza la relación entre el tamaño del grupo (díada-tríada) y la distribución argumental (simétrica-asimétrica) en lectura de tablas de doble entrada, y su relación con el tipo de respuesta utilizada. Se utilizó un diseño cuasi-experimental de solo post-test en una muestra de 89 estudiantes universitarios. Los resultados muestran distribuciones argumentales simétricas en las díadas, mientras que las tríadas obtuvieron mayores valores de asimetría. No se encontraron relaciones significativas entre el tamaño del grupo y el tipo de respuesta final como así tampoco entre la distribución argumental y el tipo de respuesta. Esto indicaría que el tamaño del grupo afecta la posibilidad de las personas de aportar argumentos durante la interacción, así como el tipo de tarea condicionaría el tipo de argumentos empleados.

Palabras clave: Argumentación, tamaño del grupo, simetría, asimetría, lectura de tabla.

Artículo de investigación. Cómo citar: Curcio, J. M., Peralta, N., \& Castellaro, M. (2019). Tamaño del grupo, argumentación y lectura de tablas en estudiantes universitarios. Diversitas: Perspectivas en Psicología, 15(2), 211-220. DOI: 10.15332/22563067.4350

Correspondencia: Dra. Nadia S. Peralta, Investigadora CONICET, Instituto Rosario de Investigaciones en Ciencias de la Educación (IRICE-CONICETUNR). Facultad de Psicología (UNR). Facultad de Psicología (UNR). Dirección postal: 27 de febrero 210 bis (Ocampo y Esmeralda), Rosario, Santa Fe, Argentina (C.P. 2000). Correo electrónico: nperalta@irice-conicet.gov.ar 


\section{Abstract}

Argumentation is an important variable to understand the learning process. Despite there are several papers about it, its relation with the group size is a novelty. The relationship between the group size (dyad-triad) and the argument distribution (symmetric-asymmetric) in a double-entry table reading task, in addition to its relationship with the type of answer used, are analyzed. A quasi-experimental design was used with only a post-test, in a sample of 89 university students. The results show that the dyads obtained the highest values of symmetry, whereas the triads obtained higher values of asymmetry. No significant relationships were found between the group size and the final answers types, neither between the group size and the answer type. This would indicate that the size of the group affects the possibility of people to provide arguments during the interaction, as well as the type of task that would condition the type of arguments used.

Keywords: Argumentation, group size, symmetry, asymmetry, table reading.

\section{Introducción}

\section{Argumentación}

El estudio de la argumentación como mediadora del aprendizaje ha cobrado relevancia en los últimos tiempos dado que constituye una habilidad requerida y valorada en los futuros profesionales. Según Baker (1998), la argumentación es una forma de interacción en donde los participantes proponen argumentos para sostener sus proposiciones $y$ contraargumentos para refutarlas. El objetivo es sostener la veracidad o probar la falsedad de éstas. También puede ser entendida como la manifestación del pensamiento o razonamiento mismo de la persona (Resnick, Salmon, Zeitz, Wathen, \& Holowack 1993; Rips, 2002).

Otro modo de definirla es según aspectos estructurales en determinado contexto. En el caso de un contexto científico y escolar, se considera al argumento como una herramienta para construir conocimiento a partir de la recopilación de fundamentos y evidencias sobre sus proposiciones, al mismo tiempo, que se utilizan refutaciones para poner a prueba su veracidad (Erduran, Simon, \& Osborne, 2004; García-Mila, Gilabert, Erduran, \& Felton, 2013). Según Billig (1989) la importancia de la argumentación recaería en la relación del individuo con el contexto, puesto que la posibilidad de sostener su postura sobre un tema específico puede depender del papel que cumple el sujeto en determinado contexto.
En síntesis, existen diversas dimensiones de la argumentación que pueden ser evaluadas conjuntamente o por separado. En primer lugar, la dimensión dialéctica, en donde la argumentación es vista como un juego verbal en el cual los participantes deben proponer distintos argumentos para sostener sus tesis. Segunda la dimensión retórica, en donde se evalúa la eficacia del argumento para convencer a las personas. Tercera la dimensión epistémica, en donde se trata del origen del argumento cuando este se considera válido para determinado campo disciplinario o socio institucional. Cuarta la dimensión conceptual, en donde el eje esta puesto en la diferenciación de los conceptos utilizados debido a sus significados, y finalmente, la dimensión interaccional, en donde se estudian las transformaciones que los argumentos pueden acontecer debido a las reglas propias de un tipo de interacción determinada (Baker, 1999).

Khun y Udell (2003) diferencian la argumentación como producto y como proceso. Lo primero implica la construcción individual de un argumento para sostener una proposición y lo segundo refiere a un proceso dialógico donde los participantes debaten sobre sus preposiciones. En esta línea, Baker (1999) también distingue la argumentación como producto de una interacción argumentativa donde habría una primera fase, en la cual los participantes proponen sus tesis y reconocen la existencia de un conflicto verbal. Luego, una segunda fase en donde proponen sus argumentos sobre dichas tesis y finalmente una fase de cierre, donde llegan a las conclusiones. 


\section{Argumentación y tablas de frecuencia}

Roselli, Dominino y Peralta (2010) y Castellaro y Roselli (2014) distinguen al menos, dos tipos de tareas cuando se trata de un trabajo colaborativo o cooperativo. Por un lado, las tareas cerradas o lógicas y, por otro, las tareas libres o abiertas. En el primer caso se encuentran, por ejemplo, los ejercicios matemáticos o la resolución de problemas, mientras que en el segundo, las discusiones dilemáticas o las actividades lúdicas.

Cuando las tareas implican colaboración, específicamente en tareas abiertas o libres, los argumentos utilizados apuntan a un consenso, mientras que en las tareas cerradas se establece una interacción cooperativa en donde cada participante busca convencer al otro (Roselli et al., 2010). Por otra parte, también se ha propuesto una relación teórica entre la calidad de los argumentos de la díada y la forma en que dicha argumentación es incentivada por el investigador. Por ejemplo, García-Milá et al., (2013) concluyeron que la calidad argumental es mayor cuando los compañeros de díada son convocados a argumentar para generar un consenso, en comparación con el argumentar para persuadir al alter. Así mismo, la existencia de un conflicto de perspectivas estimula un aumento en la calidad del discurso (Khun \& Udell, 2003; Mugny, Perret-Clermont, \& Doise, 1981).

Con respecto al presente trabajo, el mismo se enfoca en un tipo específico de tarea: las tablas de frecuencia. Éstas constituyen herramientas importantes en la educación universitaria, por su función de organización sistemática de datos. Esta organización se constituye en un formato representacional específico de organización gráfica, en la cual se entrecruza información cualitativa (categorías) y cuantitativa (frecuencias numéricas), con base en un doble eje horizontal y vertical, que permiten ordenar y sistematizar datos relacionados entre sí (Gabucio, Martí, Enfedaque, Gilabert, \& Konstantinidou, 2010). Este tipo de sistema de representación externa (Martí, 2003) es transmitido socialmente sobre todo en la educación formal, puntualmente a fines del nivel primario y durante el nivel secundario. A pesar de esto, Marti et al., (2011) detectaron problemas en la construcción de tablas en alumnos de quinto, sexto, séptimo y octavo año escolar. Según los autores, como la tabla requiere de asociaciones entre dos variables, los alumnos deberían ser capaces de organizar la información presentada dado que sería una capacidad adquirida alrededor de los 8 años cuando se ingresa al estadio operatorio concreto (Piaget \& Inhelder, 1967). Sin embargo, esto no sucede, por lo que es posible que se requiera de un andamiaje cultural que permita la apropiación de esa herramienta externa que representa la tabla (Marti et al., 2011).

Al ser el adulto quien tiene el papel de transmitir la cultura, en esta misma línea, Tauber (2010) insiste en que son los docentes los que deben tener la responsabilidad sobre los alumnos para que adquieran estas competencias, sobre todo las referidas a la estadística. En este sentido, Wu y Krajcik (2006) observan que cuando los alumnos de séptimo grado son acompañados por sus maestros en el proceso de aprendizaje de construcción de tablas y gráficos adquieren las capacidades para realizarlos. Asimismo, la construcción de tablas puede estimular a la discusión de la información a utilizar, permitiendo la construcción y negociación de significados (Peralta \& Castellaro, 2018).

\section{Tamaño del grupo}

La existencia de un conflicto cognitivo producto de la interacción con un compañero (Mugny et al., 1981) suscita a la modificación de las estructuras cognitivas, gracias al descentramiento requerido para comprender el punto de vista del otro (Piaget, 1972). Siendo así, el conflicto es predictor de desarrollo argumental (Erduran et al., 2004, García-Mila et al., 2015, Khun \& Udell, 2003) ya que la discrepancia de perspectivas estimula en los participantes la producción de argumentos que pueden llevar a la búsqueda de un consenso, o bien, a la búsqueda de convencer al otro participante de la interacción. Por este motivo, resulta importante enfocar estos trabajos en marcos interactivos (por ejemplo, díadas), ya que la presencia de un interlocutor es una condición necesaria para la génesis del conflicto sociocognitivo.

Investigaciones de estas últimas décadas han encontrado variaciones en cuanto al efecto del ta- 
maño del grupo sobre la resolución de tareas y la capacidad de colaboración de los sujetos como se ejemplifica a continuación. Capraro y Barcelo (2015), en tareas de toma de decisión dilemática grupal, encontraron que la actitud cooperativa aumenta considerablemente con grupos de 3, 5, 10 y 15 personas mientras que disminuye en grupos muy numerosos (60, 80 y 100 personas), disminuyen en cuanto a sus actitudes cooperativas.

En otro estudio, Peralta y Roselli (2017) hallaron que las díadas constituyen un escenario proclive para una interacción donde se producen resoluciones argumentativas individuales, mientras que las tríadas, utilizan más resoluciones argumentativas dialógicas. En otras palabras, las primeras tienden a buscar convencer con sus argumentos a su compañero mientras que las segundas apuntan a un consenso.

\section{Simetría-Asimetría}

El concepto de simetría (o asimetría) refiere a la igualdad (o diferencia) entre las competencias individuales específicas de los compañeros de trabajo. La relevancia de este concepto radica en que, en el marco de una tarea colaborativa que implica una demanda cognitiva particular, se afirma que la similitud o diferencia entre las competencias específicas de los sujetos influye sobre las características de la interacción y por consiguiente, en la posibilidad de beneficio cognitivo individual derivado (Castellaro \& Roselli, 2018). Según Mugny et al. (1981), para que se produzca aprendizaje sería necesario un conflicto de perspectivas mas no una gran asimetría en cuanto a las estructuras cognitivas de los participantes. Tudge $(1989,1992)$ y Asterhan et al., (2014), en problemas de resolución lógica y matemática, resaltan la importancia de una distancia cognitiva considerable entre los participantes para que se produzca un avance cognitivo en uno de ellos. Estos investigadores encuentran que se produce un avance cognitivo en el participante de menor nivel cognitivo, cuando un sujeto más avanzado interactúa con el primero.

En el presente trabajo también se considera la simetría y la asimetría entre los compañeros de trabajo, pero en relación con la contribución argumental de éstos en la realización colectiva de la tarea. Como se deriva de lo anterior, también se propone evaluar la relación entre dicha distribución argumental y el tipo de respuesta final propuesta para la solución de la actividad. En este contexto, el objetivo del presente trabajo es analizar la relación entre el tamaño del grupo y la distribución argumental en la lectura de tablas de frecuencia por parte de alumnos universitarios. Específicamente se pretende analizar la relación entre el tamaño del grupo (díada-tríada) y la distribución argumental (simetría-asimetría). En segundo lugar, entre el tamaño del grupo (díadas-tríadas) y tipo de respuesta final (lógica-social-mixta) y finalmente, entre la distribución argumental (simetría-asimetría) y el tipo de respuesta final (lógica-social-mixta).

\section{Método}

\section{Diseño}

Se empleó un diseño cuasi-experimental de sólo post-test en el que la variable independiente fue el tamaño de grupo y las variables dependientes fueron la distribución argumental (simetría/asimetría) durante la realización de la tarea y el tipo de respuesta final (lógica, social, mixta).

Para evaluar el tamaño de grupo se construyeron dos condiciones experimentales: el primer grupo trabajó en díadas y el segundo en tríadas, de acuerdo a cómo se detalla en procedimiento.

\section{Muestra}

La muestra estuvo constituida por 89 alumnos, todos ellos estudiantes de primer año de la Facultad de Psicología de la Universidad Nacional de Rosario. De este total, 56 trabajaron en 28 díadas y 33 en 11 tríadas; edad promedio de 18 años y el $90 \%$ fueron mujeres. Los grupos fueron constituidos a partir de los resultados obtenidos por los sujetos en una actividad que constituyó una medida previa para la composición de las unidades grupales, asegurando heterogeneidad de puntos de vista. La intención básica de armar este tipo de unidades sociales (con heterogeneidad interna en los puntos de vista de sus integrantes) se fundamenta en que constituye una situación sociocognitiva básica de conflicto, para el favorecimiento de la argumentación. 


\section{Materiales}

Se diseñó una actividad que consistió en la lectura de tablas de doble entrada. Este material fue constituido ad hoc, siendo utilizado como medida previa para la composición de unidades grupales y como tarea colaborativa posterior que realizaron las díadas y tríadas. Las tablas $f$ de doble entrada con valores de frecuencia y referían a investigaciones ficticias sobre fenómenos sociales donde se describían los valores obtenidos (en frecuencias) y las variables asociadas. La tabla 1 muestra una de las tablas utilizadas:

\section{Procedimiento}

Las sesiones experimentales consistieron en situaciones sociales de colaboración cognitiva en díadas y tríadas. En primer lugar, los sujetos realizaron la actividad de manera individual con el objetivo de detectar los tipos de resolución. A partir de los resultados, se constituyeron las 28 díadas conformadas cada una por dos sujetos con formas diferentes de resolver la actividad y las 11 tríadas conformadas por tres sujetos, dos de ellos con una misma forma de resolver la actividad y uno con una resolución opuesta.

Luego, tuvo lugar la situación experimental propiamente dicha, donde los sujetos de las díadas y tríadas realizaron las actividades (idénticas a la que realizaron individualmente). La consigna de trabajo solicitó a los participantes que dialogaran con el fin de llegar a una posición común relativa a la resolución de la tarea, tratando de llegar a un consenso, sin que esto fuera obligatorio. Todas las sesiones de grupos fueron grabadas para su posterior análisis.

\section{Análisis de los datos}

La variable independiente, es decir, el tamaño del grupo estuvo constituida por dos modalidades: (a) díada: dos personas y (b) tríada: tres personas. La variable dependiente "distribución argumental" refirió a la igualdad o diferencia entre la cantidad de mensajes argumentales emitidos por cada uno de los participantes a lo largo de la interacción. Se detectaron dos modalidades: (a) simétrica: cuando la cantidad de mensajes argumentales emitidos por los sujetos entre sí era igual o difería en 2 o menos unidades; (b) asimétrica: cuando la diferencia de mensajes argumentales emitidos entre sí era de más de 2 unidades. En caso de que uno de los participantes no emitía ningún mensaje y el otro sí (para el caso de las díadas), o para el caso de que un o unos participantes no emita ningún mensaje y el o los otros sí (para el caso de las tríadas), la interacción fue considerada directamente como asimétrica. Es decir, si en las díadas, por ejemplo, un participante producía 3 mensajes y su compañero 2 , la distribución argumental era considerada simétrica. En el caso de las tríadas, si uno de los participantes emitía 7 argumentos, otro 5 y otro 2, la distribución era considerada asimétrica ya que entre 5 y 2 la distancia es más amplia que 2 argumentos, aun sí entre 7 y 5 la distancia es de 2. Esto debido a que el objetivo fue describir la posibilidad que cada participante tuvo de argumentar sobre la tarea. En un contexto tríadico, si uno de los participantes no tuvo la posibilidad de argumentar en absoluto o si lo

Tabla 1.

Ejemplo de las tablas de doble entrada utilizadas durante las tareas de discusión.

\begin{tabular}{|c|c|c|c|c|}
\hline \multirow{3}{*}{$\begin{array}{l}\text { Grado de convencionalismo } \\
\text { en la vestimenta }\end{array}$} & \multicolumn{4}{|c|}{ Sexo } \\
\hline & \multicolumn{2}{|c|}{ Femenino } & \multicolumn{2}{|c|}{ Masculino } \\
\hline & $\begin{array}{c}\text { Estudiante de } \\
\text { secundario }\end{array}$ & $\begin{array}{c}\text { Estudiante } \\
\text { universitario }\end{array}$ & $\begin{array}{l}\text { Estudiante de } \\
\text { secundario }\end{array}$ & $\begin{array}{c}\text { Estudiante } \\
\text { universitario }\end{array}$ \\
\hline Convencional & 80 & 80 & 160 & 30 \\
\hline No convencional & 20 & 120 & 40 & 70 \\
\hline Total & 100 & 200 & 200 & 100 \\
\hline Total & \multicolumn{2}{|c|}{300} & \multicolumn{2}{|c|}{300} \\
\hline
\end{tabular}

Fuente: elaboración propia. 
hizo muy por debajo de la cantidad de argumentos de los otros dos participantes, la distribución argumental se entendió como asimétrica.

Se tomó como indicador de un argumento al mensaje emitido por la persona que contenía, al menos, una variable de la tabla y una justificación sobre el empleo de esa variable; por ejemplo: "la superstición es creer en cosas no empíricas", "la educación aumenta al mismo tiempo que la superstición disminuye" "cuando el estudiante se independiza tiene más capacidad de elegir la ropa que se quiere poner" "porque acá tenés 60 supersticiosos contra 90".

En cuanto a la segunda variable dependiente, el "tipo de respuesta", se definió operacionalmente como el tipo de respuesta que los sujetos dieron en consenso. La misma presentó tres modalidades: (a) lógica: cuando la respuesta expresó las causas de las variaciones de los valores de las variables

"Concluimos que el rendimiento escolar de un chico varía según la escolaridad de la madre. En el caso en que no esté escolarizada, su rendimiento es más bajo y empeora si la madre no permanece en el hogar. En los casos de madre escolarizada, no solo el rendimiento del niño es mejor si no que no influye la permanencia de ella en el hogar" (b) social: cuando la respuesta no expresó las variaciones de los valores de las variables sino que enuncia una reflexión social sobre el fenómeno ("Es normal que un estudiante secundario lleve un uniforme pactado por la institución a diferencia del universitario que se pone lo que quiere"), (c) mixta: cuando la respuesta contenía ambas características, tanto lógica como social ("Aumenta, tanto en el sexo masculino como femenino, la convencionalidad de personas que se visten de manera no convencional en el nivel universitario. Consideramos, por una cuestión de generar personalidad")

\section{Resultados}

Se encontró una relación entre el tamaño del grupo y la distribución argumental (Tabla 2). Las díadas presentaron una distribución argumental mayormente simétrica ( $75 \%$ ) mientras que en las tríadas predominó la distribución asimétrica (63.6\%). Estos resultados fueron estadísticamente significativos $\left(x^{2}=5.123 ; p<.05\right)$. Los datos obtenidos indican que los participantes en díadas producen una cantidad de argumentos más equilibrada entre sí, en comparación con las tríadas, en donde la cantidad de argumentos proporcionada por los participantes varió entre sí.

Tabla 2.

Tamaño del grupo y distribución argumental

\begin{tabular}{llccc}
\hline & & \multicolumn{3}{c}{ Distribución argumental } \\
\hline & & Asimétrica & Simétrica & Total \\
\hline \multirow{2}{*}{ Tamaño del grupo } & $7(25 \%)$ & $21(75 \%)$ & $28(100 \%)$ \\
\cline { 2 - 5 } & Diada & $7(63.6 \%)$ & $4(36.4 \%)$ & $11(100 \%)$ \\
\hline \multirow{2}{*}{ Total } & & & $25(64.1 \%)$ & $39(100 \%)$ \\
\hline
\end{tabular}

Fuente: elaboración propia.

Tabla 3.

Tamaño del grupo y tipo de respuesta

\begin{tabular}{lccccc}
\hline \multicolumn{5}{c}{ Lipo de respuesta } \\
\hline Tamaño del grupo & Díada & $18(64.3 \%)$ & $4(14.3 \%)$ & $6(21.4 \%)$ & $28(100 \%)$ \\
\cline { 1 - 2 } & Tríada & $7(63.6 \%)$ & $1(9.1 \%)$ & $3(27.3 \%)$ & $11(100 \%)$ \\
\hline Total & & $25(64.1 \%)$ & $5(12.8 \%)$ & $9(23.1 \%)$ & $39(100 \%)$ \\
\hline
\end{tabular}

Fuente: elaboración propia. 
Por otra parte, en la relación entre tamaño de grupo y tipo de respuesta, no se encontró una relación estadísticamente significativa $\left(x^{2}=0.284 ; p\right.$ $>.05$ ) entre el tamaño de grupo (díada o tríada) y el tipo de respuesta empleada (lógica, social o mixta). Se observó que, tanto en las díadas como en las tríadas, la mayor parte de las respuestas registradas fueron de carácter lógico $(64.3 \%$ y $63.6 \%$, respectivamente). Por el contrario, los dos tipos de respuestas restantes (social y mixta) mostraron proporciones menores en comparación con las lógicas (en díadas, $14.3 \%$ social y $21.4 \%$ mixta; en las tríadas $9.1 \%$ social y $27.3 \%$ mixta; ver Tabla 3 ).

Esta distribución del tipo de respuesta en díadas y triadas era esperable, dado que la tarea de lectura de tabla implicaba un proceso cognitivo por parte de los participantes donde debían asociar las variables según la variación de las frecuencias, proceso que se esperaba que se expresara en los argumentos propuestos.

En cuanto a la distribución argumental y el tipo de respuesta final, no se encontraron diferencias estadísticamente significativas $\left(x^{2}=0.487 ; p>05\right)$. Los grupos con una argumentación simétrica (64.1\%) utilizaron en un $68 \%$ respuestas del tipo lógico, $12 \%$ social y $20 \%$ del tipo mixto; mientas que los grupos asimétricos (35.9\%) utilizaron en un $57.1 \%$ respuestas del tipo lógico, $14.3 \%$ social y en un $28.6 \%$ del tipo mixto (Tabla 4).

Sin embargo, en un análisis descriptivo al interior de los grupos pudo observarse que, en las díadas los grupos que obtuvieron una distribución argumental simétrica $(75 \%)$ usaron en un $76.2 \%$ respuestas del tipo lógico, mientras que el $9.5 \%$ de las díadas utilizaron una respuesta social y $14.3 \%$ del tipo mixta, manteniendo el patrón general. Mientras que las asimétricas $(25 \%)$ usaron en un $28.6 \%$ respuestas lógicas, $28.6 \%$ social y $42.9 \%$ del tipo mixto. Es decir, en las díadas asimétricas hubo un predominio de respuestas mixtas en contraposición a los parámetros generales que indicarían uso de respuesta lógica. En las tríadas, los grupos que obtuvieron una distribución argumental simétrica (36.4\%) usaron en el $25 \%$ de los casos respuestas del tipo lógico, $25 \%$ del tipo social y $50 \%$ del tipo mixto, demostrando un predominio de este último tipo de respuesta. En cambio, las asimétricas $(63.6 \%)$ utilizaron en un $85.7 \%$ respuestas lógicas, $0 \%$ sociales y en $14.3 \%$ del tipo mixto. En síntesis, si se considera la distribución simétrica o asimétrica en general, puede observarse que el tipo de respuesta más frecuente en ambos casos es la lógica, seguida por la mixta y en menor medida la social. Ahora bien, cuando se cruzó esta variable (simetría/asimetría) con el tamaño del grupo, se observaron diferencias tanto en las díadas asimétricas como en las tríadas simétricas en donde se emplea un mayor número de respuestas mixtas para resolver la tarea.

\section{Discusión}

El objetivo del trabajo fue analizar la relación entre el tamaño del grupo y la distribución argumental en la lectura de tablas de frecuencia por parte de alumnos universitarios. En cuanto al tamaño del grupo y la distribución argumental se pudo observar que el trabajo en díadas se asocia con una distribución simétrica de argumentos, a diferencia de las

Tabla 4.

Distribución argumental y tipo de respuesta

\begin{tabular}{llcccc}
\hline & & \multicolumn{4}{c}{ Tipo de respuesta } \\
\hline Distribución argumental & Simétrica & $17(68 \%)$ & $3(12 \%)$ & $5(20 \%)$ & $25(100 \%)$ \\
\hline & Asimétrica & $8(57.1 \%)$ & $1(14.3 \%)$ & $3(28.6 \%)$ & $14(100 \%)$ \\
\hline Total & & $25(64.1 \%)$ & $5(12.8 \%)$ & $9(23.1 \%)$ & $39(100 \%)$ \\
\hline
\end{tabular}

Fuente: elaboración propia. 
tríadas donde se obtuvo una distribución asimétrica. Es decir, los aportes realizados por los sujetos de las diadas tienden a ser más equitativos que en el caso de las tríadas.

Estos resultados pueden no parecer congruentes con los obtenidos por Peralta y Roselli (2017), quienes concluyeron que las díadas utilizan mayor cantidad de argumentos que tienden a buscar el convencimiento de la otra persona mientras que las tríadas tienden a resoluciones argumentales mas consensuales. A primera vista esto pareciera ser así, pero la diferencia recae en que, en la presente investigación, tanto simetría como asimetría, se consideran en relación con los aportes argumentales de cada uno sin discriminar otro tipo de variable que pueda ilustrar si el tipo de interacción fue cooperativa o colaborativa. Esto significa que no se tomaron en cuenta si los participantes estaban de acuerdo o no con los argumentos de los compañeros sino más bien si aportaban argumentos o no. En este sentido, es posible concluir que la simetría no equivaldría a una relación colaborativa y la asimetría a una cooperativa o individualista.

Un resultado importante de destacar por su valor práctico es considerar que, si el objetivo es que todos los participantes de la interacción tengan una oportunidad igual de aportar argumentalmente, es posible que el trabajo en díadas sea más acorde, a diferencia de las tríadas. En futuras investigaciones, sería interesante estudiar la distribución argumental en grupos más numerosos utilizando una metodología similar a Capraro y Barcelo (2015). Esto último en el sentido de crear grupos más numerosos que díadas o tríadas, y variando dichos tamaños. De este modo se podría establecer una correlación entre dicha variabilidad, por un lado, y la distribución argumental por el otro.

En cuanto a la relación entre tamaño de grupo y el tipo de respuesta, se observó que el patrón de respuestas aportado, tanto por las díadas como por las tríadas, fue predominantemente lógico, seguido por las mixtas y finalmente, las respuestas sociales. Esto coincide con Roselli, Dominino y Peralta (2010) quienes concluyen que lo determinante a la hora de producir respuestas es el tipo de tarea. En este caso, al tratarse de tareas cerradas o lógicas es factible esperar respuestas lógicas, a diferencia de las tareas abiertas donde las respuestas tienden a ser variadas e incluso más vinculadas a aspectos sociales.

Finalmente, en cuanto a la distribución argumental y el tipo de respuesta, se pudo observar que en términos generales no hay diferencias significativas ya que, tanto en los grupos simétricos como asimétricos, hay un predominio de respuestas lógicas, seguido de respuestas mixtas y en menor medida respuestas sociales. Sin embargo, cuando se cruza la variable tamaño del grupo con la distribución muestral y tipo de respuesta, se pudo detectar una relación particular. A saber, las diadas asimétricas y las tríadas simétricas presentan mayores cantidades de respuestas mixtas. Esto indicaría que no solo el tipo de tarea determina la respuesta (a tareas lógicas respuestas lógicas), sino que el cruce con el tamaño del grupo moderaría la distribución general antes descripta.

Por otro lado, el tipo de respuesta mixta podría ser considerado el modo de respuesta más avanzado, ya que además de establecer las relaciones entre las variables presentadas en la tabla, los participantes reflexionan sobre las posibles causas de esos valores, estimulando a una discusión argumental más variada. Al mismo tiempo, este tipo de respuesta fomenta de alguna manera una actitud crítica hacia los datos presentados (Watson, 1997; citado de Arteaga et al. 2011), no en su aspecto estadístico como señalan los autores, pero al menos en cuanto al origen de los datos presentados.

Otro punto por destacar es la cantidad de respuestas de tipo social obtenidas en el estudio (12.8\%). Este tipo de respuestas evita establecer relaciones entre variables, por lo cual no sería una respuesta del todo correcta en la lectura de tablas de frecuencia. Sería interesante evaluar cuáles son los motivos de esta situación puesto que se podría estar ante alguna dificultad en la lectura de tablas como encuentran Marti et al. (2011) o Rodríguez Alveal y Salvador Rubilar (2012). 


\section{Referencias}

Arteaga, P., Batanero, C., Cañadas, G., \& Contreras, J. M. (2011). Las tablas y gráficos estadísticos como objetos culturales. Números, 76, 55-67.

Asterhan, C. S. C., Scwartz, B. B., \& Cohen-Eliyahu, N. (2014). Outcome feedback during collaborative learning: contingencies between feedback and dyad composition. Learning and Instruction, 34(1), 1-10.

Baker, M. J. (1998). Interpretaciones argumentativas y aprendizaje cooperativo. Revista del Centro de Ciencias del Lenguaje, 17, 133-167.

Baker, M. J. (1999). Argumentation and constructive interaction. Studies in Writing, 5, 179-202.

Billig, M. (1989). The argumentative nature of holding strong views: a case study. European Journal of Social Psychology, 19, 203-223.

Capraro, V. \& Barcelo, H. (2015). Group size effect on cooperation in one-shot social dilemmas II: curvilinear effect. PLOS ONE, 10(7), 1-11. DOI: 10.1371/journal.pone. 0131419

Castellaro, M. \& Roselli, N. (2014). Estudio exploratorio de la interacción colaborativa infantil en la realización de dibujos libres. Pensamiento Psicológico, 12(2), 37-56.

Castellaro, M. \& Roselli, N. (2018). Interacción sociocognitiva entre pares en situaciones simétricas y asimétricas de competencia epistémica. Revista de Psicología, 36(1), 1-18.

Erduran, S., Simon, S., \& Osborne, J. (2004). Tapping into argumentation: developments in the application of Toulmin's argument pattern for studying science discourse. Wiley Interscience, 88 , 915-933. DOI: $10.1002 /$ sce. 20012

Gabucio, F., Martí, E., Enfedaque, J., Gilabert, S., \& Konstantinidou, A. (2010). Niveles de comprensión de las tablas en alumnos de primaria y secundaria. Infancia y Aprendizaje, 22(2), 183-197.

García-Mila, M., Gilabert, S., Erduran, S., \& Felton, M. (2013). The effect of argumentative task goal on the quality of argumentative discourse. Science Education, 97(4), 497-523.

Khun, D. \& Udell, W. (2003). The development of argument skills. Child Development, 74(5), 1245-1260.

Martí, E. (2003). Representar el mundo externamente. La adquisición infantil de los sistemas externos de representación. Madrid, España: Antonio Machado.

Marti, E., García Mila, M., Gabucio, F., \& Konstantinidou, K. (2011). The construction of a double-entry table: A study of primary and secondary school students' difficulties. Eurpean Journal of Psychology of Education, 26, 215-234.

Mugny, G., Perret-Clermont, A. N., \& Doise, W. (1981). Interpersonal coordinations and sociological differences in the construction of the intellect. Progress in Applied Social Psychology, 1, 315-343.

Peralta, N. \& Castellaro, M. (2018). Interacción e Intersubjetividad: Investigando sus beneficios en el ámbito educativo. En J. Faccendini, P. Martino, M. Sironi y M. Terrádez (comp.), Caleidoscopio. Prácticas y Clínicas Psi en la Universidad (pp. 247-259). Rosario: UNR Editora.

Peralta, N. \& Roselli, N. (2017). Modalidad argumentativa en función del tipo de tarea y tamaño del grupo. Cogency, 9(2), 67-83.

Piaget, J. (1972). Psicología de la inteligencia. Buenos Aires, Argentina: Psique (Año de publicación del original: 1947).

Piaget, J. \& Inhelder, B. (2015). Psicología del niño. Madrid, España: Morata (Año de publicación del original: 1967).

Resnick, L. B., Salomon, M., Zeitz, C. M., Wathen Haley, S., \& Holowchak, M. (1993). Reasoning in conversation. Cognition and Intruction, $11,347-364$.

Rips, L. J. (2002). Circulate reasoning. Cognitive Science, 26, 767-795. 
Rodríguez Alveal, F., \& Sandoval Rubilar, P. R. (2012). Habilidades de codificación y descodificación de tablas y gráficos estadísticos: un estudio comparativo en profesores y alumnos de pedagogía en enseñanza básica. Avaliação, 17(1), 207-235.

Roselli, N., Dominino, M., \& Peralta, N. (2010). Influencia del tipo de tarea sobre la interacción colaborativa en equipos virtuales. Revista de Psicología General y Aplicada, 63(1), 97-117.

Tauber, L. M. (2010). Análisis de elementos básicos de alfabetización estadística en tareas de interpretación de gráficos y tablas descriptivas. Ciencias Económicas, 8(1), 53-74.
Tudge, J. (1989). When collaboration leads to regression: some negative consequences of sociocognitive conflict. European Journal of Social Psychology, 19, 123-138.

Tudge, J. (1992). Processes and consequences of peer collaboration: A vygotskian analysis. Child Development, 63, 1364-1379.

Wu, H. K., \& Krajcik, J. S. (2006). Inscriptional practices in two inquiry-based classrooms: a case study of seventh graders' use of data tables and graphs. Journal of Research in Science Teaching, 43(1), 63-95. 